\title{
Evaluation Des Performances Du Modele Agro- Hydrologique SWAT à Reproduire Le Fonctionnement Hydrologique Du Bassin Versant Nakhla (Rif occidental, Maroc)
}

\section{Roukia Boukhari Taleb (Étudiante Doctorat)}

Département des Ressources Naturelles et Environnement, Institut Agronomique et Vétérinaire Hassan II, Rabat, Maroc

\section{Pr. Mustapha Naimi \\ Pr. Mohamed Chikhaoui}

Département des Ressources Naturelles et Environnement, Institut Agronomique et Vétérinaire Hassan II, Rabat, Maroc

\section{Dr. Damien Raclot}

Institut de Recherche pour le Développement, UMR LISAH, Montpellier,France

Institut Agronomique et Vétérinaire Hassan II, Rabat, Maroc

\section{Pr. Mohamed Sabir}

Ecole Nationale Forestière d'Ingénieurs Salé, Maroc

Doi:10.19044/esj.2019.v15n5p311 URL:http://dx.doi.org/10.19044/esj.2019.v15n5p311

Résumé

Une compréhension de toutes les composantes du bilan hydrique est indispensable pour raisonner les mesures de gestion rationnelle et durable de la ressource en eau. Malheureusement, plusieurs composantes du bilan hydrique ne sont pas facilement mesurables et le recours à la modélisation est souvent nécessaire. Pour ce faire, le modèle SWAT 2012 (Soil and Water Assessment Tool) a été mis en œuvre à un pas de temps mensuel et journalier pour évaluer sa capacité à représenter le fonctionnement du bassin versant Nakhla, situé à 20 kilomètres au sud de la ville de Tétouan. Le traitement préalable des données existantes sur le bassin (MNT, pédologie, images satellitaires, données climatiques) a permis de reproduire les caractéristiques naturelles du terrain à une résolution spatiale adaptée aux objectifs de l'étude. La méthodologie adoptée repose sur l'usage du système d'information géographique libre (QGIS) combiné avec le modèle agrohydrologique SWAT pour effectuer des opérations d'analyse et de modélisation du fonctionnement hydrologique à l'échelle du bassin versant et l'outil SWAT-Cup pour optimiser les opérations d'analyse de sensibilité et 
d'incertitude. Le processus de calibration est réalisé aux échelles temporelles mensuelle et journalière au niveau de la station Timezouk et au cours de trois périodes: de 1980 à 1983 pour l'initiation, de 1984 à 1987 pour la calibration et de 1988 à 1990 pour la validation. Les résultats obtenus après calage et validation à l'échelle mensuelle montrent une simulation satisfaisante des débits puisque la comparaison des débits mensuels observés et simulés pour la période de calibration a abouti à un NSE de 0,83 et un R2 de 0,88 au niveau de la station Timezouk. Les résultats à l'échelle journalière ne sont pas satisfaisant $(\mathrm{NSE}<0.5)$, ce qui montre que la représentation de la dynamique haute-fréquence des flux d'eau doit encore être améliorée.

Mots-clés : QGIS, SWAT, hydrologie, Nakhla, Rif Occidental, Maroc

\title{
Performance Evaluation of the Agro-Hydrological SWAT Model to Reproduce the Hydrological Functioning of the Nakhla Watershed (Western Rif, Morocco)
}

\section{Roukia Boukhari Taleb (Étudiante Doctorat)}

Département des Ressources Naturelles et Environnement, Institut Agronomique et Vétérinaire Hassan II, Rabat, Maroc

\section{Pr. Mustapha Naimi}

Pr. Mohamed Chikhaoui

Département des Ressources Naturelles et Environnement, Institut Agronomique et Vétérinaire Hassan II, Rabat, Maroc

\section{Dr. Damien Raclot}

Institut de Recherche pour le Développement, UMR LISAH, Montpellier,France

Institut Agronomique et Vétérinaire Hassan II, Rabat, Maroc

Pr. Mohamed Sabir

Ecole Nationale Forestière d'Ingénieurs Salé, Maroc

\begin{abstract}
A comprehension of all the components of water balance is essential for rational and durable management of the water resource. However, the direct assessment of several components of the water balance is very difficult and the use of modeling is often necessary. SWAT 2012 (Soil and Water
\end{abstract}


Assessment Tool) model was thus implemented on a monthly and daily time steps to assess his ability to reproduce the hydrological functioning for the Nakhla watershed, which is located at 20 kilometers in the south of the city of Tetouan. Treatment of environmental data (MNT, pedology, satellite imagery and climatic data) allowed to describing the natural characteristic of the field at a resolution adapted to the objectives of the study. Adopted methodology consisted in the combined use of the SWAT agro hydrological model implemented in the QGIS geographical information system open source for operations of analysis and modeling of hydrological processes at the level of the watershed area and of SWAT-CUP tool to optimize the operations of sensitivity and uncertainty analysis. The calibration process was performed on two temporal scales; monthly and daily; and on the stations Timezouk; and over three periods: warm-up period from 1980 to 1983, calibration period from 1984 to 1987, and the validation period from 1988 to 1990 . The results acquired after calibration and validation at monthly time step were satisfactory and presented good level of realism in the simulation of the discharge. The comparison of the monthly observed and simulated discharges for the period of calibration gave a NSE of 0.83 and R2 of 0.88 at the Timezouk station. Nevertheless, daily results are not satisfactory $(\mathrm{NSE}<0.5)$, thus further improvements are required to better simulate the processes at a finer time step.

Keywords: QGIS, SWAT, hydrology, Nakhla, Western Rif, Morocco

\section{INTRODUCTION:}

Le développement durable des activités humaines est basé, en particulier, sur l'approvisionnement en eau. La gestion intégrée de l'eau, nécessaire pour accompagner un développement durable qui concilie développement et conservation de l'environnement, consiste notamment à assurer l'eau en quantité et en qualité suffisantes pour les besoins humains (eau potable, industrielle, irrigation, etc.) et environnementaux. Elle doit également tenir compte des événements extrêmes, sécheresses et inondations dont la fréquence pourrait s'accroitre avec le changement climatique. Cette gestion ne peut être efficace que lorsqu'elle est fondée sur une maîtrise des processus qui contrôlent les variations et la disponibilité de l'eau à une échelle donnée. Comprendre les processus impliqués dans le devenir de l'eau météorique est essentiel pour prédire la disponibilité de cette ressource et par la suite envisager son exploitation.

$\mathrm{Au}$ Maroc, en raison du contexte climatique et hydrologique difficile, la gestion rationnelle de l'eau est primordiale. Les précipitations sont dominées par une forte irrégularité dans l'espace et dans le temps, l'alternance des années de haute hydraulicité et de sécheresse grave est une caractéristique 
des régimes climatiques et hydrologiques du Maroc. En effet, bien qu'ayant des chaines de montagnes importantes, bordé par l'océan Atlantique à l'Ouest et la mer Méditerranée au Nord et doté d'une diversité des eaux superficielles (lacs, rivières, etc.) et souterraines, ces ressources en eau restent limitées par rapport à la forte demande résultante de la croissance démographique, l'amélioration des conditions de vie, le développement des industries et l'extension de l'irrigation. Actuellement le potentiel en eau varie de $180 \mathrm{~m}^{3} / \mathrm{hab} /$ an pour les zones réputées très pauvres en ressources en eau, situées au sud du Maroc ; à près de $1850 \mathrm{~m}^{3} / \mathrm{hab} / \mathrm{an}$ pour les bassins situés au nord ayant des ressources en eau abondantes (Bzioui, 2004). D'ici 2020, les ressources hydriques par habitant à travers le pays seraient d'environ 720 $\mathrm{m}^{3} /$ hab/an (Bzioui, 2004) et $35 \%$ de la population totale du pays disposeraient de moins de $500 \mathrm{~m}^{3} / \mathrm{hab} / \mathrm{an}$ (Bzioui, 2004).

Afin de résoudre le dilemme de gestion de l'eau, une bonne maîtrise des différents processus hydrologiques s'impose. Évidemment, cette maîtrise doit être effectuée à l'échelle du bassin versant comme étant l'unité géographique de base pour l'analyse du cycle hydrologique et de ses composantes. Les processus hydrologiques et leurs variabilités spatiales sont contrôlés par la topographie, la géologie et l'utilisation des terres au niveau $\mathrm{du}$ bassin versant (Ghoraba, 2015). Afin de simuler des processus hydrologiques à l'échelle du bassin versant, plusieurs modèles hydrologiques distribués prenant en compte la relation multifactorielle entre les conditions naturelles et le fonctionnement hydrologique ont été développés. On peut citer MODFLOW (McDonald and Harbaugh, 1988), TOPMODEL (Beven and KIirkby, 1979), SHE (Abbott et al., 1986), MODCOU (Ledoux et al., 1989), SWAT(Arnold et al., 1998) et MGB-IPH (Collischonn and Tucci, 2001). Parmi ces modèles, SWAT s'est montré le plus performant à diverses reprises pour reproduire le fonctionnement hydrologique des bassins versants (Pereira et al., 2016).

SWAT a été appliqué dans le monde entier sur une grande diversité d'utilisations des terres (Zhang et al., 2016). Il a également été testé à différentes échelles, allant de la parcelle cultivée au bassin versant et même à l'échelle de la région (Arnold et al., 2000). Il a été utilisé pour des finalités très diverses telles que l'étude de la gestion de bassin versant (Santhi et al., 2001; Tuppad et al., 2010; Meaurio et al., 2015), la modélisation des activités agricoles (Van Liew et al., 2003; Srinivasan et al., 2010; Meaurio et al., 2015), ou encore l'étude de l'effet des changements climatiques sur le fonctionnement hydrologique (Zhang et al., 2015).

Seules quelques études de modélisation par SWAT ont été réalisées au Maroc (Briak et al., 2016). Ceci dit, les thématiques étudiées sont diversifiées: l'adéquation au milieu de haute montagne avec des apports de précipitations sous forme de neige (Chaponniere et al., 2003), l'évaluation de 
l'impact de gestion de terre dans le bassin de Sebou (Terink et al., 2011), la modélisation des débits (Fadil et al., 2011; Kharchaf et al., 2013), l'érosion des sols (Briak et al., 2016), la modélisation spatiale de la recharge d'eaux souterraines en couplant les modèles SWAT et MODFLOW (Chkara and El Morabiti, 2016). À travers sa recherche dans le cadre de thèse, Brirhet (2016) a démontré une meilleure robustesse de SWAT par rapport aux modèles HEM-HMS et ATHYS en matière de reproduction du fonctionnement hydrologique du bassin versant marocain d'Issen.

Le besoin d'une meilleure planification et gestion des ressources en eau pour la ville de Tétouan est la raison pour laquelle nous avons décidé d'effectuer cette étude. Bien que les précipitations annuelles de la ville de Tétouan soient importantes, la forte saisonnalité de celles-ci résulte dans de fréquentes coupures d'approvisionnement en eau potable de la ville. Et malgré, les sources qui surgissent de la dorsale calcaire et qui alimentent la rivière de Martil, ainsi que les eaux stockées au niveau du barrage Nakhla, assurer la continuité d'alimentation en eau potable de la ville de Tétouan reste un défi non relevé. Dans l'objectif d'identifier les solutions possibles, cette étude met en œuvre le modèle SWAT dans le bassin versant Nakhla. Cette mise en œuvre s'appuie sur une description détaillée de la topographie, de la géologie, de la pédologie et de l'occupation du sol du bassin. Des étapes de paramétrisation, de calibration et de validation du modèle permettent ensuite de tester la capacité du modèle SWAT à reproduire le fonctionnement hydrologique du bassin versant Nakhla à travers l'évaluation de la performance de reproduction de deux termes importants du bilan hydrique dont l'écoulement et l'évaporation.

\section{MATÉRIELS ET MÉTHODES:}

\section{Zone d'étude :}

Le bassin versant Nakhla (figure 1) d'une superficie de $110,8 \mathrm{~km}^{2}$ est situé à $20 \mathrm{Km}$ au sud de la ville de Tétouan. Il est caractérisé par un relief accidenté, avec une altitude allant de $1800 \mathrm{~m}$ au niveau des sommets calcaires dans le sud-est du bassin, à $160 \mathrm{~m}$ au niveau du barrage et par une pente supérieure à $20^{\circ}$ pour plus de $50 \%$ de la superficie du bassin. Les données climatiques mettent en relief le caractère méditerranéen du bassin, une saison sèche allant du mois de mai-juin à septembre et une saison pluvieuse allant d'octobre à avril. Les précipitations annuelles sont de 670 $\mathrm{mm}$ au niveau de la station du barrage durant la période 1980-2008. Les formations géologiques sont dominées par deux ensembles de roches aux caractéristiques contrastées: une dorsale calcaro dolomitique dure et compacte sur une formation marno-schisteuse tendre (Naimi et al., 2003). La géologie combinée aux autres facteurs de pédogenèse ont permis la formation d'une couverture pédologique dominée par les sols minéraux 
bruts, les sols peu évolués, les sols calcimagnésiques et les sols à sesquioxydes de fer ou de manganèse. L'agriculture dans le bassin versant est une agriculture traditionnelle en majorité pluviale. Les zones irriguées sont limitées aux terrasses installées à proximité des sources qui surgissent au niveau de la dorsale calcaire. La production agricole est faible et destinée surtout à l'autoconsommation sauf pour la culture du tabac qui est destinée au marché. La végétation naturelle est dominée par le chêne liège, les lauriers et le tamaris.

\section{Description du modèle SWAT :}

Le modèle SWAT est le résultat de plus de 30 années de développement au niveau du département de l'agriculture et des laboratoires de l'université de Texas A \& M (Krysanova and White, 2015) aux Etats Unis. Le modèle SWAT est adopté dans la présente étude du fait de son application réussie à travers le monde. De plus, son caractère « Open source » ouvre des horizons d'adaptation du modèle à d'éventuelles spécificités du milieu étudié. Dans cette étude, le modèle SWAT est mis en œuvre à travers l'interface QGIS dédiée, QSWAT (Version 2012), ce qui facilite la préparation des données d'entrée et la visualisation des sorties de SWAT (Dile et al., 2016).
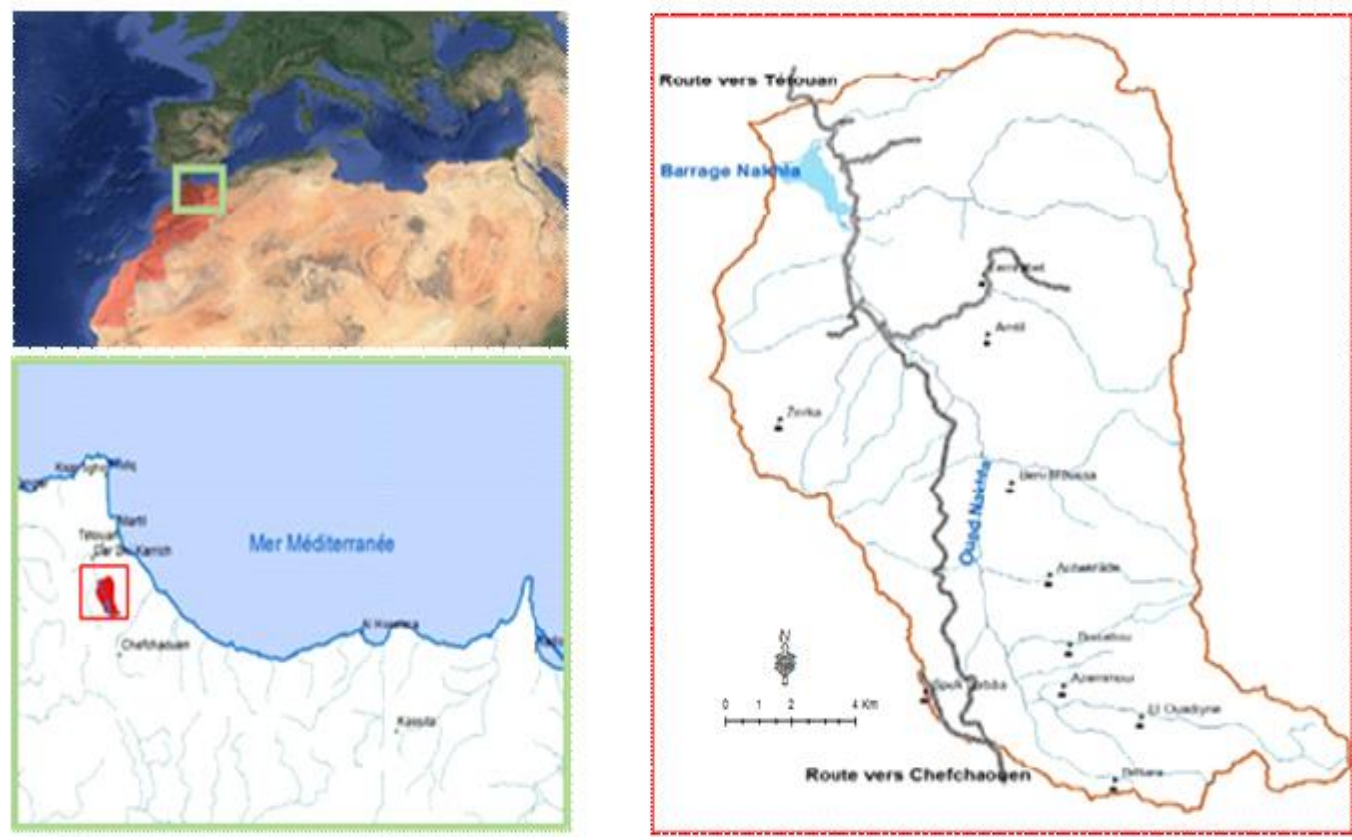

QSWAT est un modèle continu, semi-distribué, orienté processus (Arnold et al., 1998; Gassman et al., 2007; Pereira et al., 2016) qui fonctionne principalement à un pas de temps journalier et mensuel. Il englobe des modules hydrologiques, chimiques, écologiques, de croissance de plantes et prend en compte les pratiques d'aménagement. Il permet de 
simuler le développement des cultures, les flux hydriques dans tous les compartiments (eau, sol, plantes, etc. ...), ainsi que les flux de sédiments et d'éléments nutritifs à grandes et petites échelles ( $\mathrm{Lu}$ et al., 2015; Sinnathamby et al., 2017). Aussi, il permet la prédiction des impacts à long terme de gestion agricole et d'utilisation des terres sur l'eau (Arnold et al., 2012).

La modélisation du fonctionnement hydrologique a lieu en deux étapes : le bassin versant est tout d'abord divisé en sous-bassins, reliés par un réseau hydrographique, puis chaque sous-bassin est divisé en unités de réponse hydrologiques (URH), chacune contenant une combinaison unique de pente, d'utilisation des terres, de type de sol et des pratiques de gestion représentant ainsi l'hétérogénéité spatiale (Zhang et al., 2016) du milieu étudié. Les URH sont les unités de base au niveau desquelles le modèle calcule le bilan hydrique, la croissance des cultures et l'effet des pratiques et aménagements culturaux tels que l'application d'engrais ou d'aménagement antiérosif. Le ruissellement de chaque sous-bassin est évalué en prenant en compte le bilan hydrique sur l'ensemble des URH qui le composent, ce qui permet d'évaluer la contribution en ruissellement du sous bassin au réseau hydrographique.

La modélisation du bilan hydrique; adoptée par SWAT; prend en compte quatre compartiments de stockage: la neige, le profil du sol (0-2 m) l'aquifère peu profond $(2-20 \mathrm{~m})$ et l'aquifère profond $(>20 \mathrm{~m})$ (Bilondi et al., 2013). Meaurio (2015) a synthétisé les différentes fonctions adoptées par SWAT afin de modéliser le processus hydrologique : le ruissellement est prédit pour les précipitations journalières en utilisant la méthode SCS modifiée (USDASCS, 1972), le débit de pointe est calculé avec la méthode rationnelle modifiée (Chow et al., 1988), l'écoulement souterrain latéral dans le profil du sol (0-2 m) est déterminé dans chaque couche de sol avec le modèle cinématique de stockage (Sloan et al., 1983) la contribution de l'écoulement des eaux souterraines au débit total est simulée par la création de réservoirs aquifères peu profonds (Arnold and Allen, 1999) et la percolation du fond de la zone racinaire est considérée comme une recharge vers l'aquifère peu profond, le flux au sein du réseau de drainage de surface est acheminé en utilisant la méthode du coefficient de stockage variable (Williams, 1969).

\section{Outil d'analyse de sensibilité :}

SWAT-CUP (SWAT Calibration and Uncertainty Procedures) regroupe un ensemble de procédures de calibration et d'analyse d'incertitudes pour le modèle SWAT à travers une seule interface afin de rendre la calibration plus facile (Abbaspour et al., 2007). Cinq approches sont proposées : SUFI2, GLUE, ParaSol, MCMC et PSO.

Sequential Uncertainty Fitting (SUFI-2) est adopté dans cette étude car il permet une flexibilité élevée dans le choix des paramètres, des intervalles, de 
l'échelle de temps et les sous-bassins à calibrer (Abbaspour et al., 2007; Bilondi et al., 2013; Malagò et al., 2015). Dans SUFI-2, l'identification des paramètres sensibles est effectuée à l'aide des indicateurs t-stat et P-value. Un paramètre est plus sensible lorsqu'il a une valeur absolue élevée de t-stat et une valeur de P-value proche de zéro.

\section{Outils d'évaluation des performances du modèle :}

La performance du modèle SWAT est évaluée en premier lieu graphiquement, ensuite en utilisant deux indices statistiques. Le premier, Nash-Sutcliffe Efficiency NSE indique à quel point la variation des observations correspond à la variation des valeurs simulées (équation 1). Il varie de $-\infty$ à 1 avec 1 comme valeur optimale qui traduit la superposition des données simulées et observées. Lorsque la valeur de NSE est supérieure à 0,5 , les résultats obtenus sont considérés satisfaisants. Le second est le coefficient de détermination $\mathrm{R}^{2}$ (équation 2 ). Il représente la proportion de variance expliquée dans la variance totale des observations. Sa valeur varie de 0 (modèle nul) à 1 (modèle parfait), avec des résultats considérés satisfaisant lorsqu'il dépasse 0,5 (Green et al., 2006).

Equation 1: NSE $=1-\frac{\sum(O-S)^{2}}{\sum(O-\bar{O})^{2}} \quad$ Equation2: $R^{2}=\frac{\left[\sum(O-\bar{O}) \cdot(S-\bar{S})\right]^{2}}{\left[\sum(O-\bar{O})\right] \cdot[\Sigma(S-\bar{S})]^{2}}$

Avec $O$ les valeurs observées ; $\bar{O}$ la moyenne des valeurs observées ; $S$ les valeurs simulées et $\bar{S}$ la moyenne des valeurs simulées.

\section{Alimentation du modèle :}

Trois sources d'incertitudes associées aux sorties de modèles sont généralement différenciées : les incertitudes structurelles propres au modèle, les incertitudes liées au calage des paramètres non observables et les incertitudes sur les données d'entrées ou de contrôle (Shrestha et al., 2016). Les deux premières sont propres au modèle et aux procédures de calage utilisées. L'incertitude sur les données d'entrée et de contrôle peut par contre être minimisée grâce à l'expertise sur la qualité des données existantes en relation avec une connaissance du milieu naturel acquise par les prospections de terrains.

Les principales données contextuelles exigées par le modèle SWAT sont : la topographie, la pédologie (caractéristiques physiques et chimiques du sol), l'utilisation du sol et les données climatiques. Les données spatiales ont été gérées sous QGIS. Les données utilisées pour la modélisation du bassin Nakhla sont les suivantes : 
Un Modèle numérique de terrain (MNT) produit par une image Aster (Taille de la cellule : $20 \times 20 \mathrm{~m}$ ), corrigé afin d'éliminer les cuvettes. Il permet par la suite de délimiter le bassin versant et les sous bassins, les chenaux d'écoulement, et dériver la carte des pentes.

Une carte des sols (figure 5) réalisée à une échelle de 1:50000. La couverture pédologique est organisée en 26 classes. Chaque classe possède un profil type décrit sur le terrain, avec une série d'analyses pour chaque horizon : granulométrie, matière organique. Cette connaissance a permis de construire la base de données des sols de Nakhla composé d'un ensemble de 14 paramètres pour chaque profil. Le facteur d'érodabilité des sols (USLE_K) est estimé par l'équation de Barril (1988) adopté par (Ait Brahim et al., 2002), la conductivité hydraulique à saturation SOL_K, et la capacité de stockage du sol, SOL_AWC sont estimées par les équations de pédotransfert proposées par Wosten et al.(1999) établies sur la base de données des sols HYPRES (Van Den Bogaert, 2011).

Une carte d'occupation des sols réalisée grâce à une classification supervisée d'une image satellitaire Landsat ETM 1991, considérant six classes dominantes : terrain de cultures, végétation naturelle dégradée, matorral dense, matorral clair, barrage Nakhla et la dorsale calcaire.

Les données météorologiques: Les précipitations sont reproduites à partir de deux stations (Nakhla, Timezouk) dont la localisation est présentée au niveau du tableau (1). La température est renseignée par les mesures journalières minimales et maximales à partir de la station météorologique de l'aéroport de Tanger. Les radiations solaires, l'humidité relative ainsi que la vitesse du vent sont déduites à partir des moyennes mensuelles. Toutes ces données ont été intégrées dans la base de données climatique SWAT afin de pouvoir simuler l'évapotranspiration moyennant la formule de PenmanMonteith. Les données ont été fournies par l'Agence du Bassin Hydraulique Loukkos (ABHL) et par le centre météorologique de l'aéroport TANGER. 


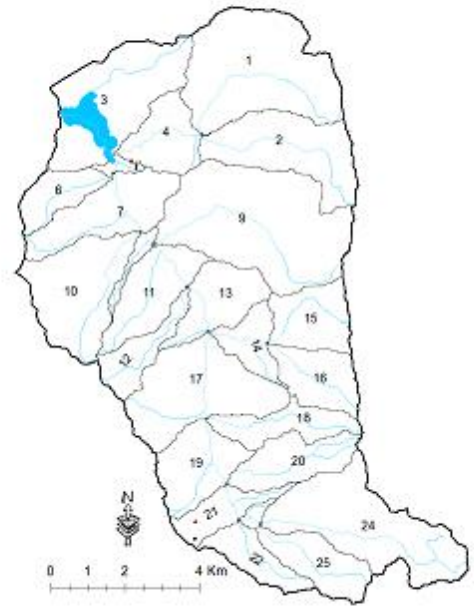

Figure 2 Carte des sousbassins

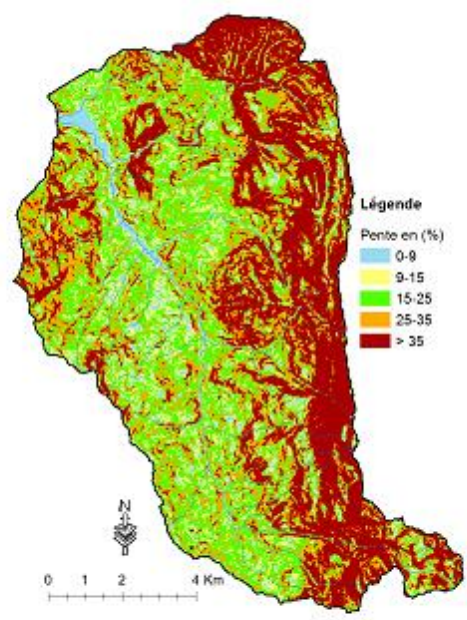

Figure 4 Carte des classes de pente

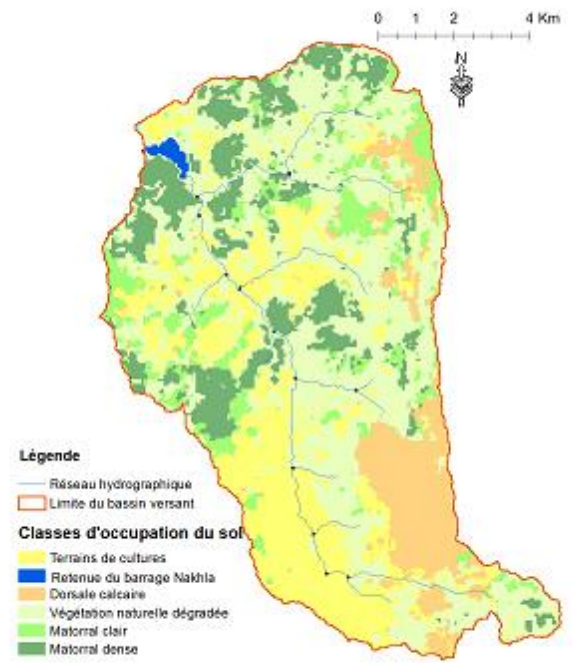

Figure ${ }^{3 A}$ Carte d'occupation des sols

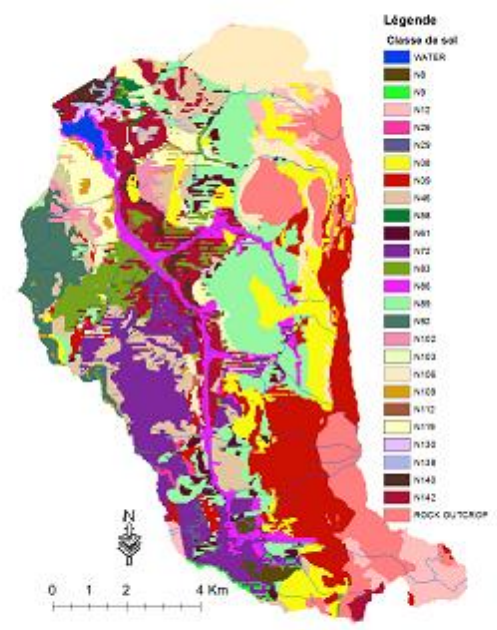

Figure 5. Carte des classes de sol

Les données de contrôle: Les mesures de débits journaliers à l'échelle de la station de mesure Timezouk, les débits journaliers au niveau du barrage Nakhla, ainsi que l'évaporation mesurée par la méthode de Bac installé au niveau de la station barrage Nakhla, sont obtenues à partir de données mesurées par l'Agence de Bassin Versant Loukkos pour la période 1980 1990. 
Tableau 1: localisation des stations de mesure de pluie

\begin{tabular}{|c|c|c|c|c|}
\hline $\mathrm{D}$ & Nom de la station & Latitude (XPR) & Longitude (YPR) & Elévation \\
\hline 1 & Nakhla & 499100 & 538100 & 210 \\
\hline 2 & Timezouk & 502600 & 532500 & 290 \\
\hline
\end{tabular}

\section{Délimitation du bassin versant :}

Le modèle numérique de terrain est utilisé pour tracer les chemins et les sens d'écoulement dans un système d'information géographique open source (QGIS). Un seuil de 200 ha est adopté pour définir l'origine du réseau hydrographique. Un exutoire est imposé au niveau de la station de mesure Timezouk et un second au niveau de la retenue du barrage qui contrôle la rivière de Nakhla. Le bassin versant est subdivisé en 25 sous-bassins (figure 2). Dans le bassin versant Nakhla, les types d'utilisation du sol sont regroupés en sept classes basées sur les espèces dominantes (figure 3) alors que le sol est classé en 26 types (figure 5). Les grilles de pente (figure 4) sont reclassées sur la base du MNT en cinq classes de pentes (0-9\%, 9-15\%, 15 $25 \%, 25-35 \%$ et $>35 \%$ ). Les couches de végétation, de sol et de pente sont fusionnées pour créer les unités de réponse hydrologique (HRU) dans chaque sous-bassin. Un total de 706 HRU est identifié dans le bassin versant Nakhla.

\section{Analyse de sensibilité, calibration et validation :}

L'analyse de sensibilité des paramètres est une méthode d'identification des changements des variables de sortie en fonction des changements des paramètres du modèle ( $\mathrm{Lu}$ et al., 2015). Afin de déterminer les paramètres sensibles, une analyse de sensibilité approfondie a été mise en place au niveau de la station Timezouk. Celle-ci s'est appuyée sur l'approche One-ata-time de SWAT-CUP pour évaluer la sensibilité de chaque paramètre individuellement. Une analyse globale a ensuite été effectuée pour prendre en compte l'effet des interactions entre paramètres (Zhang et al., 2016). Dans cette étude, la période 1980 - 1983 a été utilisée pour l'initialisation du modèle, la période 1984 à 1987 comme période de calibration et celle 19881990 comme période de validation. La procédure adoptée pour la calibration du modèle SWAT au niveau du bassin versant Nakhla s'est déroulée en plusieurs étapes successives. Elle a débuté par une calibration automatique puis manuelle à l'échelle mensuelle. La calibration est ensuite affinée en s'appuyant sur les mesures journalières mesurées au niveau de la station Timezouk. La validation mensuelle est mise en place à l'échelle de la station Timezouk et Nakhla alors que la validation journalière est effectuée seulement au niveau de la station Timezouk. 


\section{RÉSULTATS ET DISCUSSION}

\section{Résultats du modèle SWAT avant calibration :}

Dans la mesure où le modèle SWAT est élaboré sur la base des caractéristiques des sols, de la végétation et de l'hydrologie des bassins Nord-américain, l'analyse des résultats de simulation avant calibration permet de voir à quel point le modèle SWAT reproduit le fonctionnement hydrologique du bassin versant Nakhla avec les données d'entrées (MNT, classe de pente, sol, occupation des terres, précipitation) et les règles de calibration utilisées par défaut.

L'application sans calibration du modèle SWAT à un pas de temps mensuel donne des résultats moyennement satisfaisants avec 0,54 et 0,47 pour $\mathrm{R}^{2}$ et NSE respectivement et, $0,73 \mathrm{~m}^{3} / \mathrm{s}$ comme moyenne des débits simulés et $1,12 \mathrm{~m}^{3} / \mathrm{s}$ comme moyenne des débits observés au niveau de la station de mesure Timezouk au cours de la période allant de 1983 à 1990. Au niveau de la station de barrage Nakhla la qualité des simulations est du même ordre, avec $\mathrm{R}^{2}$ et NSE égales respectivement à 0,58 et 0,56 et un débit moyen simulé de $1,69 \mathrm{~m}^{3} / \mathrm{s}$ pour un débit moyen observé de $1,56 \mathrm{~m}^{3} / \mathrm{s}$, ceci illustre la capacité de SWAT à simuler l'écoulement mensuel au niveau de la station de barrage Nakhla. Cette capacité de reproduction sans calage constitue un bon indicateur de la qualité des données d'entrée (MNT, pédologie, utilisation des terres, climat) utilisées.

Cependant, les résultats sont non satisfaisants à l'échelle journalière avec NSE de $-0,80$ et $\mathrm{R}^{2}$ de 0,18 . Ainsi, la calibration journalière s'impose afin de pouvoir reproduire la variabilité du fonctionnement hydrologique à un pas de temps journalier qui est fortement lié à l'aléa climatique.

\section{Résultats de l'analyse de sensibilité :}

Les résultats de l'analyse de sensibilité pour le bassin versant Nakhla (récapitulés dans le Tableau 2) approuve la qualité des inputs utilisés qui représentent d'une façon optimale la réalité du terrain. Les paramètres les plus sensibles sont liés aux paramètres que les données de base (pédologie, occupation du sol, pente, climat) ne peuvent pas renseigner et auxquels le modèle attribut des valeurs par défaut. Dont les paramètres physiques des canaux qui contrôlent le ruissellement de surface : l'encombrement végétatif (rugosité de Manning : $\mathrm{CH}_{-} \mathrm{N}$ ) qui influence le temps de concentration et par la suite la dynamique de crue, la conductivité hydraulique du lit des cours d'eau $\left(\mathrm{CH}_{-} \mathrm{K}\right)$ qui contrôle les échanges entre la rivière et la nappe. Les paramètres qui régissent le transfert des eaux souterraines ne sont pas à négliger, par défaut une grande partie des eaux s'écoule vers la nappe au moment où la nappe profonde est absente au niveau du bassin versant Nakhla, ainsi les paramètres qui régissent les échanges d'eau avec la nappe avait un grande influence sur la qualité de reproduction du fonctionnement 
hydrologique. Le premier paramètre est RCHRG-DP qui détermine la proportion d'eau qui migre de l'aquifère peu profond à l'aquifère profond par percolation et permet de faire varier la quantité d'eau qui quitte les couches superficielles. Le second est GW-DELAY qui détermine le décalage entre le temps où l'eau sort du sol et entre dans l'aquifère peu profond. Le troisième est ALPHA-BF, la constante de récession qui est la réponse à la recharge de la nappe. Et en fin GWQMN, la quantité d'eau qui reste dans l'aquifère sans jamais se vider.

Certains paramètres qui déterminent les proportions évaporées à partir de différents compartiments sont aussi sensibles. L'évapotranspiration est évaluée pour chaque jour par l'évaporation des plantes en fonction de la période de croissance et l'évaporation des sols. Ce dernier processus est calibré par le facteur de compensation de l'évaporation des sols ESCO; et par les facteurs GW-REVAP et REVAPMN qui déterminent la quantité d'eau permettant une remontée capillaire d'eau de l'aquifère peu profond jusqu'à la zone non saturée. GW-REVAP est un paramètre restrictif de ce flux au moment où REVAPMN est le volume seuil d'eau dans l'aquifère peu profond en dessous duquel les mouvements d'eau par capillarité sont inexistants.

\section{Résultats de la calibration et la validation :}

Après calibration des paramètres les plus sensibles (Tableau 2), l'analyse des indices statistiques NSE et $\mathrm{R}^{2}$ montre que les simulations d'écoulement sont satisfaisantes. Pour la période ayant servie à la calibration, les valeurs pour NSE et $R^{2}$ ont respectivement de 0,83 et 0,88 (figure 6) au niveau des débits mensuels à la station Timezouk. Les indices évalués à l'échelle mensuelle pour la période de validation sont de 0.77 et 0,80 (figure 7) pour NSE et $\mathrm{R}^{2}$ au niveau de la station Timezouk. Au pas de temps journalier, les valeurs NSE et $\mathrm{R}^{2}$ sont respectivement à 0,48 et 0,63 pour la période de calibration et à 0,43 et 0,74 pour la période de validation au niveau de la station Timezouk (figure 9).

Les résultats du calage mensuel sont jugés satisfaisants (NSE $>0,5)$. Par contre les résultats au pas de temps journalier (calage et validation) ne sont pas satisfaisants, avec un indice NSE inférieur à 0,5 qui reflète une capacité limitée à reproduire les fluctuations des débits (figure 8 et 9). Certes, le calage arrive à reproduire la variation générale des débits simulés, comme l'indique d'assez bons coefficients de détermination, mais une sousestimation des débits, surtout de pointe persiste malgré la procédure de calage effectuée. 
Tableau 2 : Résultat de l'analyse de sensibilité réalisée par SWAT-Cup

\begin{tabular}{|c|c|c|c|c|c|c|}
\hline Paramètre & $\begin{array}{c}\mathbf{P}- \\
\text { value }\end{array}$ & T-stat & Définition & Fichiers & \begin{tabular}{|c|} 
Intervalle \\
a
\end{tabular} & \begin{tabular}{|c|} 
Valeur \\
adoptée $^{b}$
\end{tabular} \\
\hline $\mathrm{Ch}-\mathrm{K} 2$ & 0 & 9.21 & $\begin{array}{l}\text { Conductivité hydraulique effective dans } \\
\text { l'alluvion du canal principal }(\mathrm{mm} / \mathrm{h})\end{array}$ & $\begin{array}{l}\text { Sous- } \\
\text { bassin } \\
(. r t e)\end{array}$ & $0-150$ & 143.53 \\
\hline Ch-N2 & 0 & 6.06 & $\begin{array}{l}\text { Le Coefficient de Manning "n" du canal } \\
\text { principal }\end{array}$ & $\begin{array}{l}\text { Sous- } \\
\text { bassin } \\
(. r t e)\end{array}$ & $0.02-0.2$ & 0.127 \\
\hline Gwqmn & 0.03 & -2.14 & $\begin{array}{l}\text { Hauteur d'eau minimale pour qu'il y ait } \\
\text { un transfert d'eau de l'aquifère peu } \\
\text { profond vers le cours d'eau }\end{array}$ & $\begin{array}{l}\text { Sous- } \\
\text { bassin } \\
(. g w)\end{array}$ & $0-500$ & 1.54 \\
\hline $\begin{array}{l}\text { GW-REV } \\
\text { AP }\end{array}$ & 0.49 & -0.67 & $\begin{array}{l}\text { Coefficient permettant le transfert d'eau } \\
\text { de l'aquifère vers les horizons sus- } \\
\text { jacents et non saturés du sol }\end{array}$ & $\begin{array}{c}\text { Bassin } \\
(. b s n)\end{array}$ & $0.02-0.2$ & 0.15 \\
\hline Gw-Delay & 0.55 & -0.58 & $\begin{array}{l}\text { Délai pour que l'eau souterraine qui } \\
\text { transite par l'aquifère peu profond } \\
\text { atteigne le cours d'eau (jours) }\end{array}$ & $\begin{array}{l}\text { Sous- } \\
\text { bassin } \\
(. g w)\end{array}$ & $0-40$ & 22.6 \\
\hline Alpha bf & 0.23 & 1.19 & $\begin{array}{l}\text { Constante de récession de l'écoulement } \\
\text { de base souterrain }\end{array}$ & $\begin{array}{l}\text { Sous- } \\
\text { bassin } \\
(. g w) \\
\end{array}$ & $0-1$ & 0.56 \\
\hline ESCO & 0.30 & -1.033 & $\begin{array}{l}\text { Facteur de compensation d'évaporation } \\
\text { du sol }\end{array}$ & $\begin{array}{c}\text { Bassin } \\
(. b s n)\end{array}$ & $0.01-1$ & 0.7 \\
\hline REVAPMN & 0.35 & 0.91 & $\begin{array}{l}\text { Hauteur d'eau minimale dans l'aquifère } \\
\text { peu profond pour qu'il y ait un } \\
\text { mouvement d'eau vers le sol ("revap") } \\
\text { ou l'aquifère profond }\end{array}$ & $\begin{array}{l}\text { Sous- } \\
\text { bassin } \\
(. g w)\end{array}$ & $\begin{array}{l}0.0016- \\
500\end{array}$ & 330 \\
\hline RCHRG_DP & 0.41 & 0.82 & $\begin{array}{l}\text { Taux de recharge de l'aquifère profond } \\
\text { par percolation }\end{array}$ & $\begin{array}{l}\text { Sous- } \\
\text { bassin } \\
(. g w)\end{array}$ & $0-1$ & 0.008 \\
\hline
\end{tabular}

a : Intervalle adopté pour l'analyse de sensibilité, b : Valeur adoptée pour la calibration ; (.rte) : fichier qui englobe les paramètres représentants les caractéristiques physiques du cours d'eau ;

(.gw) : fichier qui englobe les paramètres représentants les caractéristiques de la nappe ;

(.bsn) : fichier qui définit les attributs général du bassin 


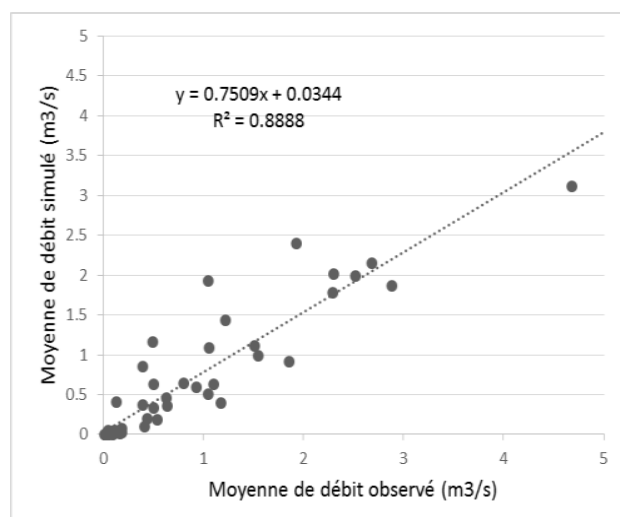

Figure 6 : Comparaison du débit mensuelle moyenne simulée et mesurée au niveau de la station Timezouk par régression linéaire, période de calibration.

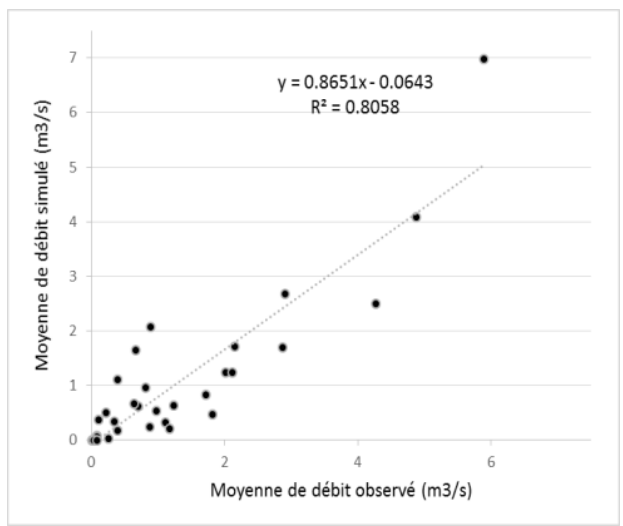

Figure 7 : Comparaison du débit mensuelle moyenne simulée et mesurée au niveau de la station Timezouk par régression linéaire, période de validation.

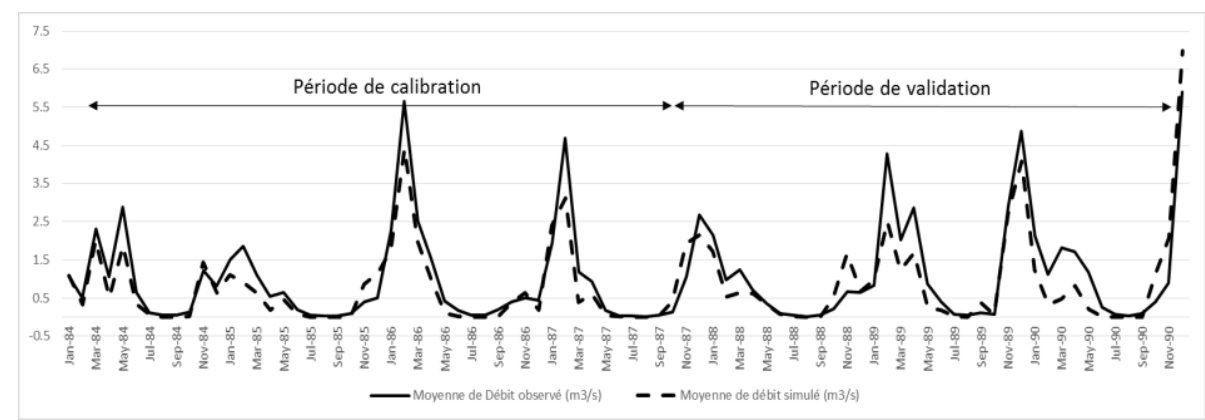

Figure 8 : Comparaison des débits mensuels observés et simulés, station Timezouk

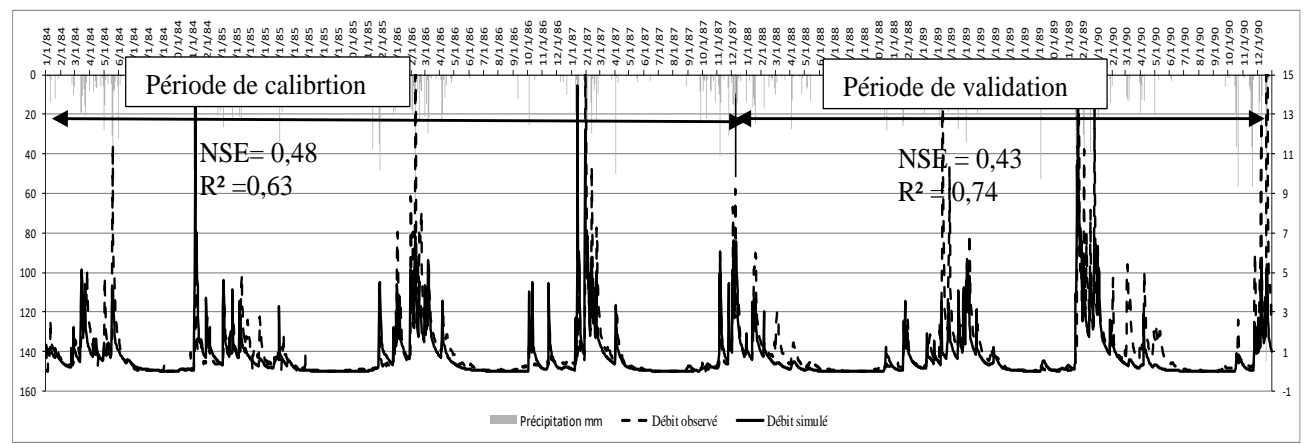

Figure 9 : Comparaison des débits journaliers observés et simulés, station Timezouk 


\section{Analyse du bilan hydrique du bassin versant Nakhla :}

L'analyse des différentes composantes du bilan hydrique permet d'affiner le diagnostic sur le fonctionnement du modèle et d'identifier les pistes d'améliorations à privilégier.

L’analyse du bilan hydrique annuel (récapitulés dans le Tableau 3) le long des périodes (1984-1987) et (1988 - 1990) montre de faibles fluctuations d'une période à l'autre, liées principalement aux conditions climatiques vu la stabilité des autres facteurs à l'échelle de temps considéré (occupation du sol, type de sol, pente et altitude). Ainsi, en moyenne $70 \%$ des pluies reçues par le bassin versant Nakhla alimentent les cours d'eau; dont environ $30 \%$ d'écoulement direct et $40 \%$ (infiltration et percolation) transitent par les horizons du sol pour rejoindre ensuite les cours d'eau ; alors que d'environ $30 \%$ des pluies retournent à l'atmosphère par évaporation au niveau du bassin de stockage et des cours d'eau et évapotranspiration.

Tableau 3: Bilan hydrologique du bassin versant Nakhla

\begin{tabular}{|l|c|c|c|c|}
\hline \multirow{2}{*}{ Moyenne annuelle } & Période de & \multirow{2}{*}{ Pourcentage } & $\begin{array}{l}\text { Période de } \\
1988 \text { à } 1987\end{array}$ & \multirow{2}{*}{ Pò 1990 } \\
\cline { 4 - 5 } & $691 \mathrm{~mm}$ & & $779 \mathrm{~mm}$ & \\
\hline Précipitation & $187 \mathrm{~mm}$ & $27 \%$ & $244 \mathrm{~mm}$ & $31 \%$ \\
\hline Ruissellement & $127 \mathrm{~mm}$ & $18 \%$ & $139 \mathrm{~mm}$ & $18 \%$ \\
\hline Infiltration & $157 \mathrm{~mm}$ & $23 \%$ & $164 \mathrm{~mm}$ & $21 \%$ \\
\hline Percolation & $221 \mathrm{~mm}$ & $32 \%$ & $232 \mathrm{~mm}$ & $30 \%$ \\
\hline Evapotranspiration & \multicolumn{3}{|l}{} \\
\hline
\end{tabular}

L'analyse des débits simulés au pas de temps journalier montre que l'écoulement est sous-estimé par SWAT. En effet le débit moyen journalier mesuré au niveau de la station Timezouk au cours de la période de calibration est de $0.9 \mathrm{~m}^{3} / \mathrm{s}$ pour un débit moyen simulé de $0,7 \mathrm{~m}^{3} / \mathrm{s}$. Les débits moyens mesurés et simulés sont respectivement de 1.2 et $0.9 \mathrm{~m}^{3} / \mathrm{s}$ pour la période de validation. Cette sous-estimation peut s'expliquer par la mauvaise adéquation du pas de temps journalier dans un contexte de crue rapide durant quelques heures seulement. Ainsi l'utilisation des moyennes journalières de pluies ne permettent pas de bien reproduire la genèse des débits de pointe. Aussi la localisation des stations ne reproduit pas les quantités de pluie reçues en altitude, vu que les stations de mesure des précipitations sont situées à des altitudes d'environ $200 \mathrm{~m}$ pour Nakhla et Timezouk alors que plus de $40 \%$ de la superficie du bassin est caractérisée par des altitudes entre $400 \mathrm{~m}$ et $1800 \mathrm{~m}$.

L'évaporation constitue une variable principale du bilan hydrologique vu qu'elle représente une part importante du bilan. Toutefois, elle est assez difficile à mesurer directement (Aouissi et al., 2016) et son calcul nécessite l'acquisition d'un grand nombre de données météorologiques.

En l'absence de mesure directe de l'évapotranspiration de chacun des couverts végétaux présentent au niveau du bassin versant Nakhla; on s'est 
contenté de tester les performances de SWAT à reproduire les exportations par évaporation à travers La comparaison (figure 10) de l'évaporation simulée par SWAT à un pas de temps mensuelle à l'échelle de la HRU représentant barrage Nakhla avec les mesures effectuées à l'échelle de la station Barrage Nakhla Moyennant la méthode de Bac d'évaporation. L'analyse de régression linéaire de l'évaporation simulés contre l'évaporation observés indiquent une corrélation de $87 \%$ (figure 11); ceci prouve la robustesse de la méthode de Penman-Monteith adoptée pour simuler l'évaporation et sa capacité à remplacer les méthodes dites directes tel que la méthode de Bac dont la mesure est largement critiquée.

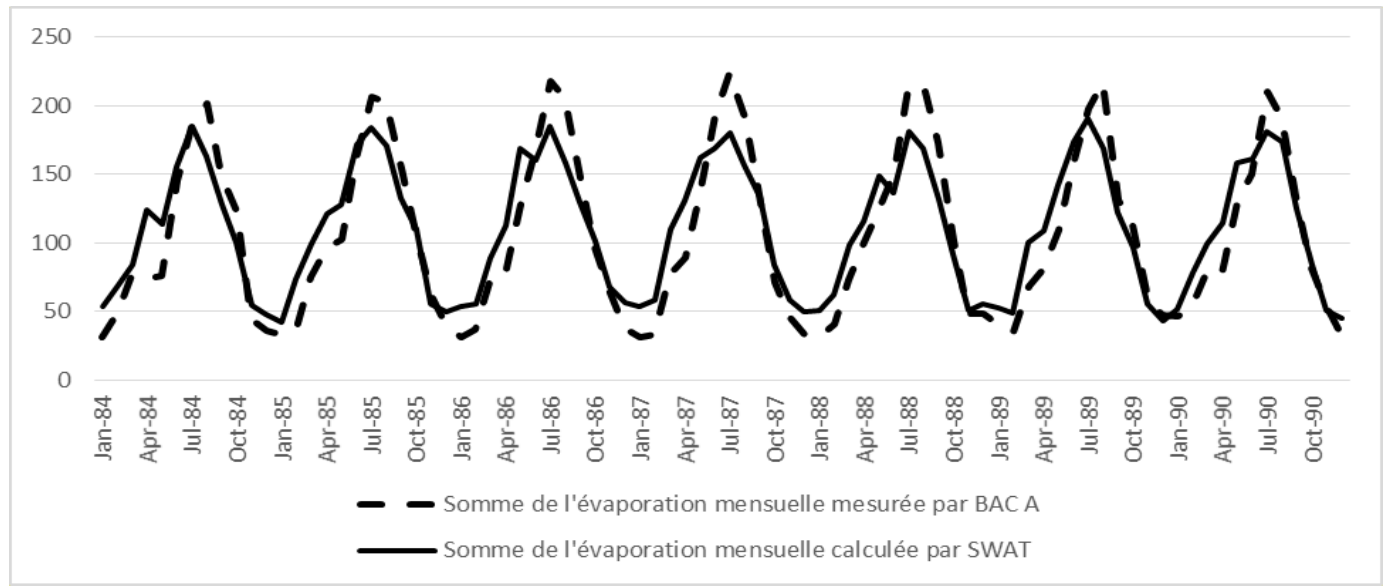

Figure 1: Comparaison de la somme de l'évaporation mensuelle mesurée par Bac et simulée par SWAT, période de calibration et validation

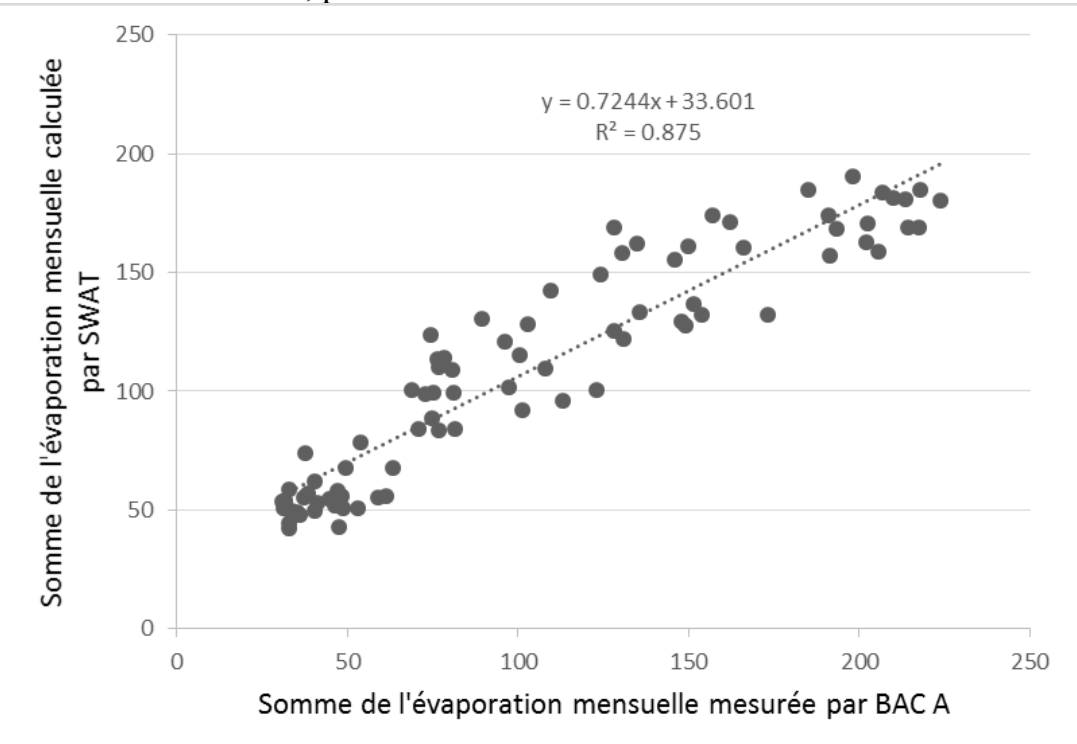

Figure 2: Comparaison de l'évaporation mensuelle moyenne mesurée par Bac et simulée au niveau du HRU-barrage Nakhla par régression linéaire. 


\section{CONCLUSION ET RECOMMANDATIONS:}

Dans cette recherche, le modèle SWAT est mis en œuvre pour la période allant de 1980 à 1990 au niveau du bassin versant Nakhla à travers une calibration multi-temporelle (mensuelle et journalière) au niveau du site Timezouk. La comparaison graphique des débits observés et simulés pendant la période de calage et de validation a permis de mettre en relief la capacité $\mathrm{du}$ modèle à reproduire de manière satisfaisante les séries de débits à l'échelle mensuelle cependant la reproduction à l'échelle journalière n'était pas de la même qualité.

La capacité de reproduction de SWAT des débits journaliers s'est montrée très limitée. Une explication est la difficulté de reproduire les débits de pointe à partir des pluies journalières moyennes dans un contexte climatique de fortes averses sur de courte durée. Le passage à l'échelle infrajournalière dans SWAT est une piste à tester dans ce contexte de crues rapides, sachant que la disponibilité de pluie à des pas de temps infrajournalier est très limitée au Maroc.

L'analyse du bilan hydrique nous a permis d'identifier les principales pistes d'améliorations qui consistent à la reproduction du gradient orographique du bassin afin de remédier à la sous-estimation des débits par le modèle. Une meilleure reproduction de la relation pluie-altitude à travers le bassin versant Nakhla permettra une meilleure reproduction des différents termes du bilan hydrologiques, ce qu'est un préalable indispensable avant de vouloir tester la composante érosion de ce modèle. Aussi l'adoption de calibration multisite, afin de déterminer l'ordre de grandeur de tous les paramètres et vérifier si la distribution spatiale des prévisions est satisfaisante (Muleta and Nicklow, 2005) et surtout améliorer les performances du modèle au niveau de la station de barrage Nakhla. La prolongation de la période de calibration dans l'objectif de prendre en considération la variabilité temporelle du fonctionnement hydrologique imposée par la variabilité climatique est aussi nécessaire pour une meilleure adaptation du modèle SWAT au bassin versant Nakhla.

Cependant, notre connaissance de la complexité du milieu naturel étudié ; le bassin versant Nakhla; permets de souligner que les résultats achevés prouvent la robustesse du modèle à reproduire les différents processus naturels interactifs et complexes ayant lieu à l'échelle du bassin versant. L'outil SWAT est alors adapté à la modélisation et à la compréhension du fonctionnement hydrologique. Il est surtout un moyen prometteur pour évaluer quantitativement l'érosion et identifier la prévalence spatiale du phénomène au niveau du bassin versant; et par la suite un instrument de bon augure pour une gestion intégrée des ressources en sol et en eau alliée à une exploitation durable au niveau du bassin Nakhla et des bassins avoisinants de caractéristiques biophysiques similaires. 


\section{REFERENCES:}

1. Abbaspour, K.C., Vejdani, M., and Haghighat, S. (2007). SWATCUP: calibration and uncertainty programs for SWAT. In International Congress on Modelling and Simulation, Modelling and Simulation Society of Australia and New Zealand.

2. Abbott, M.B., Bathurst, J.C., Cunge, J.A., O'Connell, P.E., and Rasmussen, J. (1986). An introduction to the European Hydrological System - Systeme Hydrologique Europeen, "SHE”, 2: Structure of a physically-based, distributed modelling system. Journal of Hydrology 87, 61-77.

3. Ait Brahim, L., Sossey Alaoui, F., Siteri, H., EL Hamdouni, I., Tahiri, M., and Baghdad, B. (2002). Prise en compte des glissements de terrain dans la quantification des pertes en sols dans le bassin versant Nakhla (Télédétection et SIG).

4. Aouissi, J., Benabdallah, S., Chabaâne, Z.L., and Cudennec, C. (2016). Evaluation of potential evapotranspiration assessment methods for hydrological modelling with SWAT-Application in data-scarce rural Tunisia. Agricultural Water Management 174, 39 51.

5. Arnold, J.G., and Allen, P.M. (1999). Automated methods for estimating baseflow and ground water recharge from streamflow records1. JAWRA Journal of the American Water Resources Association 35, 411-424.

6. Arnold, J.G., Srinivasan, R., Muttiah, R.S., and Williams, J.R. (1998). Large Area Hydrologic Modeling and Assessment Part I: Model Development. Journal of the American Water Resources Association 34, 73-89.

7. Arnold, J.G., Muttiah, R.S., Srinivasan, R., and Allen, P.M. (2000). Regional estimation of base flow and groundwater recharge in the Upper Mississippi river basin. Journal of Hydrology 227, 21-40.

8. Arnold, J.G., Moriasi, D.N., Gassman, P.W., Abbaspour, K.C., White, M.J., Srinivasan, R., Santhi, C., Harmel, R.D., van Griensven, A., Van Liew, M.W., et al. (2012). SWAT: Model Use, Calibration, and Validation. Transactions of the ASABE 55, 1491-1508.

9. Barril, P. (1988). Notes de cours choisies sur l'érosivité des pluies (à l'INAT) Lausanne: Ecole polytechnique fédéral de Lausanne; Institut du Génie Rural.

10. Beven, K.J., and KIirkby, M.J. (1979). A physically based, variable contributing area model of basin hydrology. Hydrological Sciences Bulletin 24, 43-69.

11. Bilondi, M.P., Abbaspour, K.C., and Ghahraman, B. (2013). Application of three different calibration-uncertainty analysis 
methods in a semi-distributed rainfall-runoff model application differential evolution adaptive metropolis algorithm. Middle-East Journal of Scientific Research 15, 1255-1263.

12. Briak, H., Moussadek, R., Aboumaria, K., and Mrabet, R. (2016). Assessing sediment yield in Kalaya gauged watershed (Northern Morocco) using GIS and SWAT model. International Soil and Water Conservation Research 4, 177-185.

13. Brirhet, H. (2016). Intégration des modèles globaux et distribués dans la modélisation hydrologique du bassin versant d'Issen, région de Souss. Thèse. Faculté des sciences et techniques, Fès.

14. Bzioui, M. (2004). Rapport national sur les ressources en eau du Maroc.

15. Chaponniere, A., Maisongrande, P., Escadafal, R., Solan, B. de, and Chehbouni, G. (2003). Satellite driven modeling of snow runoff in a small semi-arid mountainous watershed in Morocco. In IGARSS 2003. 2003 IEEE International Geoscience and Remote Sensing Symposium. Proceedings (IEEE Cat. No.03CH37477), pp. 11811183 vol.2.

16. Chkara, I., and El Morabiti, K. (2016). Evaluation de la recharge des eaux souterraines par une approche basée sur le modèle intégré eaux de surface - eaux souterraines. In 43rd IAH CONGRESS 25-29th September, 2016 Le Corum , Montpellier, France.

17. Chow, V., Maidment, D., and Mays, L. (1988). Applied hydrologySeries in water resources and environmental engineering (McGrawHill Inc., New York, NY).

18. Collischonn, W., and Tucci, C.E.M. (2001). Simulação hidrológica de grandes bacias. Revista Brasileira de Recursos Hídricos 6, 95-118.

19. Dile, Y.T., Daggupati, P., George, C., Srinivasan, R., and Arnold, J. (2016). Introducing a new open source GIS user interface for the SWAT model. Environmental Modelling \& Software 85, 129-138.

20. Fadil, A., Rhinane, H., Kaoukaya, A., Kharchaf, Y., and Bachir, O.A. (2011). Hydrologic modeling of the Bouregreg watershed (Morocco) using GIS and SWAT model. Journal of Geographic Information System 3, 279.

21. Gassman, P.W., Reyes, M.R., Green, C., and Arnold, J.G. (2007). The soil and water assessment tool: historical development, applications, and future research directions. Transactions of the American Society of Agricultural and Biological Engineers 50, 1211-1250.

22. Ghoraba, S.M. (2015). Hydrological modeling of the Simly Dam watershed (Pakistan) using GIS and SWAT model. Alexandria Engineering Journal 54, 583-594. 
23. Green, C., Tomer, M., Di Luzio, M., and Arnold, J. (2006). Hydrologic evaluation of the soil and water assessment tool for a large tile-drained watershed in Iowa. Transactions of the ASABE 49, 413-422.

24. Kharchaf, Y., Rhinane, H., Kaoukaya, A., and Fadil, A. (2013). The Contribution of the Geospatial Information to the Hydrological Modelling of a Watershed with Reservoirs: Case of Low Oum Er Rbiaa Basin (Morocco). Journal of Geographic Information System $5,258$.

25. Krysanova, V., and White, M. (2015). Advances in water resources assessment with SWAT-an overview. Hydrological Sciences Journal 60, 771-783.

26. Ledoux, E., Girard, G., De Marsily, G., Villeneuve, J.P., and Deschenes, J. (1989). Spatially distributed modeling: conceptual approach, coupling surface water and groundwater. In Unsaturated Flow in Hydrologic Modeling, H.J. Morel-Seytoux, ed. (Springer Netherlands), pp. 435-454.

27. Lu, Z., Zou, S., Xiao, H., Zheng, C., Yin, Z., and Wang, W. (2015). Comprehensive hydrologic calibration of SWAT and water balance analysis in mountainous watersheds in northwest China. Physics and Chemistry of the Earth, Parts A/B/C 79-82, 76-85.

28. Malagò, A., Pagliero, L., Bouraoui, F., and Franchini, M. (2015). Comparing calibrated parameter sets of the SWAT model for the Scandinavian and Iberian peninsulas. Hydrological Sciences Journal=.

29. McDonald, M.G., and Harbaugh, A.W. (1988). A modular threedimensional finite-difference ground-water flow model (U.S. G.P.O.,).

30. Meaurio, M., Zabaleta, A., Uriarte, J.A., Srinivasan, R., and Antigüedad, I. (2015). Evaluation of SWAT models performance to simulate streamflow spatial origin. The case of a small forested watershed. Journal of Hydrology 525, 326-334.

31. Muleta, M.K., and Nicklow, J.W. (2005). Sensitivity and uncertainty analysis coupled with automatic calibration for a distributed watershed model. Journal of Hydrology 306, 127-145.

32. Naimi, M., Tayaa, M., Ouzizi, S., Choukra-llha, R., and Kerby, M. (2003). Sécheresse. (John Libbey Eurotext Ltd).

33. Pereira, D. dos R., Martinez, M.A., Pruski, F.F., and da Silva, D.D. (2016). Hydrological simulation in a basin of typical tropical climate and soil using the SWAT model part I: Calibration and validation tests. Journal of Hydrology: Regional Studies 7, 14-37. 
34. Santhi, C., Arnold, J.G., Williams, J.R., Dugas, W.A., Srinivasan, R., and Hauck, L.M. (2001). Validation of the Swat model on a Large Rwer basin with point and nonpoint Sources. JAWRA Journal of the American Water Resources Association 37, 1169-1188.

35. Shrestha, M.K., Recknagel, F., Frizenschaf, J., and Meyer, W. (2016). Assessing SWAT models based on single and multi-site calibration for the simulation of flow and nutrient loads in the semiarid Onkaparinga catchment in South Australia. Agricultural Water Management 175, 61-71.

36. Sinnathamby, S., Douglas-Mankin, K.R., and Craige, C. (2017). Field-scale calibration of crop-yield parameters in the Soil and Water Assessment Tool (SWAT). Agricultural Water Management 180, Part A, 61-69.

37. Sloan, P.G., Moore, I.D., Coltharp, G.B., and Eigel, J.D. (1983). Modeling subsurface stormflow on steeply sloping forested watersheds. Water Resources Research 20, 1815-1822.

38. Srinivasan, R., Zhang, X., Arnold, J., and others (2010). SWAT ungauged: hydrological budget and crop yield predictions in the Upper Mississippi River Basin. Trans. ASABE 53, 1533-1546.

39. Terink, W., Hunink, J.E., Droogers, P., Reuter, H.I., J, L. van G.W., and Kauffman, J.H. (2011). Impacts of land management options in the Sebou Basin: Using the Soil and Water Assessment Tool SWAT.

40. Tuppad, P., Kannan, N., Srinivasan, R., Rossi, C.G., and Arnold, J.G. (2010). Simulation of agricultural management alternatives for watershed protection. Water Resour Manage 24, 3115-3144.

41. USDA-SCS (1972). National engineering handbook: Hydrology section 4 (US Department of Agriculture, Soil Conservation Service Washington, DC).

42. Van Den Bogaert, R. (2011). Typologie des sols du bassin versant de la Morcille, caractérisation de leurs propriétés hydrauliques et test de fonctions de pédotransfert (Université Pierre et Marie Curie (Paris 6); Institut des Sciences et Industries du Vivant et de l'Environnement).

43. Van Liew, M.W., Arnold, J.G., and Garbrecht, J.D. (2003). Hydrologic simulation on agricultural watersheds: Choosing between two models. Transactions of the ASAE 46, 1539-1551.

44. Williams, J.R. (1969). Flood Routing With Variable Travel Time or Variable Storage Coefficients. Transactions of the ASAE 12, 0100 0103 . 
45. Wosten, J.H.M., Lilly, A., Nemes, A., and Le Bas, C. (1999). Development and use of a database of hydraulic properties of European soils. Geoderma 90, 169-185.

46. Zhang, D., Chen, X., Yao, H., and Lin, B. (2015). Improved calibration scheme of SWAT by separating wet and dry seasons. Ecological Modelling 301, 54-61.

47. Zhang, D., Chen, X., Yao, H., and James, A. (2016). Moving SWAT model calibration and uncertainty analysis to an enterprise Hadoopbased cloud. Environmental Modelling and Software 84, 140-148. 